\title{
Airway inflammation is augmented by obesity and fatty acids in asthma
}

\author{
H.A. Scott*,\# , P.G. Gibson*,\#, M.L. Garg ${ }^{\star}$ and L.G. Wood ${ }^{\star, \#}$
}

ABSTRACT: Obesity and asthma are associated, but the mechanism(s) of the association have yet to be elucidated. The aim of this study was to assess airway inflammation in relation to obesity and plasma fatty acids in males and females with and without asthma.

Obese $(n=68)$ and nonobese $(n=47)$ adults with asthma, and obese $(n=16)$ and nonobese $(n=63)$ healthy controls had induced sputum and venous blood samples analysed for inflammatory markers.

There was a positive interaction between obesity and asthma on sputum neutrophil percentage $(p=0.012)$ and $C$-reactive protein level $(p=0.003)$. Although sputum eosinophil percentage was elevated in asthma $(p=0.001)$, there was no effect of obesity $(p=0.16)$. Sputum neutrophil percentage was positively associated with body mass index in females with asthma $(\beta=1.015,95 \%$

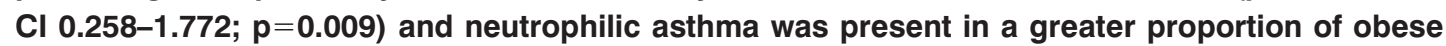
compared with non-obese females $(42.9 \%$ versus $16.2 \% ; p=0.017)$. In males with asthma, sputum neutrophil percentage was positively associated with total plasma saturated fatty acids $(\beta=0.108$, $95 \% \mathrm{Cl} 0.036-0.180 ; p=0.004)$ and negatively with monounsaturated fatty acids $(\beta=-0.068,95 \% \mathrm{Cl}$ $-0.131--0.005 ; p=0.035)$.

This was the first study to demonstrate an increase in neutrophilic airway inflammation in obese asthma. This relationship was significant only in females with asthma. In males, saturated and monounsaturated fatty acids were important predictors of neutrophilic airway inflammation in asthma.

KEYWORDS: Asthma, inflammation, leptin, neutrophils, obesity, saturated fat

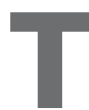
he prevalence of both asthma and obesity has increased in recent years and evidence suggests that the two conditions are associated. Although the mechanism(s) responsible for the association remain unknown, a number of hypotheses, including inflammation, gastro-oesophageal reflux and mechanical factors, have been explored [1]. Researchers have examined the role of asthmatic airway inflammation and have found no relationship with obesity, measured directly via sputum eosinophils [2-4] or indirectly by exhaled nitric oxide [4]. This has led to the general consensus that airway inflammation is not driving the association between asthma and obesity.

There is now evidence of a noneosinophilic pattern of inflammation in asthma, with a subgroup of asthmatics demonstrating interleukin (IL)-8 driven neutrophilic airway inflammation [5]. Neutrophilic asthma involves innate immune dysfunction, with a persistence of symptoms and airway hyperresponsiveness (AHR) in the absence of sputum eosinophils [5]. Three recent studies reported no relationship between obesity and neutrophilic airway inflammation; however, all showed a clear nonsignificant trend towards increased sputum neutrophils as body mass index (BMI) increased [2-4]. The lack of statistical significance in these studies is possibly due to the small sample size in two studies [2, 4], sex differences not being considered in two instances [2,3] and the large variation generally observed in the measurement of sputum cell counts. A recent cluster analysis performed by HALDAR et al. [6] identified a unique obese-asthma phenotype: a population that is obese and predominantly female, with an absence of airway eosinophils. It is possible that this noneosinophilic phenotype represents neutrophilic asthma. Indeed, neutrophils have been shown to be more highly activated in obesity, both in the circulation [7] and adipose tissue vasculature [8], indicating that the characteristic low-grade inflammation seen in obesity appears to be the result of chronic activation of the innate immune response. It is possible that this inflammation extends to the airways of susceptible individuals, leading to increased levels of airway neutrophils.

Obesity leads to a well-described increase in circulating levels of the pro-inflammatory cytokines
AFFILIATIONS

*Respiratory and Sleep Medicine, Hunter Medical Research Institute, John Hunter Hospital,

${ }^{\#}$ School of Medicine and Public Health, and

'Nutraceuticals Research Group,

School of Biomedical Sciences and Pharmacy, University of Newcastle, Newcastle, Australia.

\section{CORRESPONDENCE}

L.G. Wood

Dept of Respiratory and Sleep Medicine, HMRI, Level 3

John Hunter Hospital

Lookout Rd

New Lambton Heights NSW 2305

Newcastle

NSW

Australia

E-mail: Lisa.Wood@

newcastle.edu.au

Received:

Sept 012010

Accepted after revision:

Jan 172011

First published online:

Feb 102011 
IL-6 and C-reactive protein (CRP), due to the presence of excess adipose tissue, which is metabolically active. A high-fat diet can also lead to increased systemic inflammation, independent of BMI, due to an increase in circulating fatty acid levels, which activate innate immune responses [9]. Systemic inflammation is evident in asthma, with pro-inflammatory mediators elevated in asthmatics compared with healthy controls [10]. Therefore, markers of systemic inflammation that are elevated both in obesity and by circulating saturated fatty acids are also elevated in asthma, suggesting the independent inflammatory effects of obesity and fatty acids may contribute to the development and progression of asthma.

A number of population-based studies have suggested that the relationship between obesity and asthma may be stronger in females [11], though this is not a universal finding. A sexual dimorphism exists in relation to body composition, in that females carry more fat subcutaneously and males carry more fat viscerally [12]. This leads to distinct differences in the inflammatory pattern exhibited. For example, leptin is secreted more highly from subcutaneous adipose tissue and is therefore higher in females than males [12]. Fatty acids are also handled differently, with greater storage in females (resulting in a comparatively higher adiposity) and greater release of fatty acids into the portal circulation of males [13]. Because of the known sexual dimorphism in body composition and handling of fatty acids, and the discovery of an obese-asthma phenotype that is predominantly female [6], we sought to examine the effects of sex on the relationship between obesity, fatty acids and asthma.

We hypothesised that obesity and plasma fatty acids contribute independently to innate immune activation, leading to increased levels of airway neutrophils and systemic inflammation, with differences in the pattern of inflammation between males and females. The aim of this study was to assess airway inflammation in relation to obesity and plasma fatty acids in males and females with and without asthma.

\section{MATERIALS AND METHODS Subjects}

Subjects were obese (BMI $\geqslant 30 \mathrm{~kg} \cdot \mathrm{m}^{-2}$ ) and nonobese (BMI $<30 \mathrm{~kg} \cdot \mathrm{m}^{-2}$ ) adults with and without asthma. Asthma was defined as a doctor's diagnosis of asthma and AHR to $4.5 \%$ hypertonic saline. All asthmatic subjects were classified as stable: they had no asthma exacerbation, respiratory tract infection or oral corticosteroid use in the preceding 4 weeks. All subjects were nonsmokers. Subjects underwent skin allergy testing and asthmatic subjects completed the Asthma Control Questionnaire (ACQ) [14]. Body weight was determined using calibrated electronic scales (Nuweigh EB8271; Newcastle Weighing Services, Wickham, Australia) measuring in $0.1-\mathrm{kg}$ increments. Height was measured using a wall-mounted stadiometer (Seca 220; Seca, Hamburg, Germany), measuring to the nearest millimetre. This research was approved by the Hunter New England Human Research Ethics Committee (Hunter New England Health, New Lambton, Australia) and all subjects provided written informed consent.

\section{Sputum induction and analysis}

Spirometry (KoKo; nSpire Health, Longmont, CO, USA) and sputum induction coupled with bronchial provocation using
4.5\% hypertonic saline was performed over a standardised 15.5-min nebuliser time, as described by GIBSON et al. [15]. A decrease in forced expiratory volume in $1 \mathrm{~s}$ (FEV1) of $\geqslant 15 \%$ of baseline was indicative of AHR and was recorded as the provocation dose of saline [15]. A fall in FEV1 of $\geqslant 15 \%$ of baseline was treated with a $\beta_{2}$-agonist (salbutamol, $200 \mu \mathrm{g}$ ). Sputum samples were successfully obtained from $88 \%$ of asthmatic subjects and $82 \%$ of healthy controls.

Lower respiratory sputum portions were selected and dispersed using dithiothreitol. Total cell counts and cell viability (trypan blue exclusion) were determined using a haemocytometer. Differential cell counts were determined from cytospins, which were prepared, stained (May-Grunwald Geimsa) and counted from 400 nonsquamous cells.

\section{Serum inflammatory markers}

Venous blood was collected after a 12-h overnight fast. Commercial ELISAs were used to determine high-sensitivity CRP (MP-Biomedicals, Orangeburg, NY, USA), IL-6 (R\&D Systems, Minneapolis, MN, USA) and leptin (Bio-Rad, Hercules, CA, USA) levels. Assay sensitivities were $0.1 \mathrm{mg} \cdot \mathrm{L}^{-1}, 0.039$ $\mathrm{pg} \cdot \mathrm{mL}^{-1}$ and $13 \mathrm{pg} \cdot \mathrm{mL}^{-1}$ respectively.

\section{Plasma fatty acid analysis}

Whole blood was collected in EDTA tubes and centrifuged at $3,000 \times g$ at $4^{\circ} \mathrm{C}$ for $10 \mathrm{~min}$. The plasma was separated and stored at $-70^{\circ} \mathrm{C}$ before analysis. Total fatty acids were determined as described previously [16].

\section{Statistical analysis}

Data are reported as median (interquartile range (IQR)) for nonparametric data or mean $\pm \mathrm{SD}$ for parametric data. Group comparisons for continuous data were performed using either the Kruskal-Wallis test with post hoc Wilcoxon rank sum testing or ANOVA with post hoc two-sample unpaired t-testing (Intercooled Stata 9.1; Stata Corporation, College Station, TX, USA). Group comparisons of proportions were compared using Pearson's Chi-squared test. Sputum neutrophil percentages were analysed by backward and forward stepwise regression until the most parsimonious model was achieved. Univariate $\mathrm{p}$-values of $\leqslant 0.2$ entered the multivariate model. These analyses were conducted in asthmatic subjects as a whole, asthmatic females and asthmatic males. Age- and sexadjusted systemic and airway inflammation was analysed by two-factor ANOVA in asthmatic and nonasthmatic subjects, to determine the interactions and main effects of asthma and obesity. The robust variance estimator was applied to nonparametric variables to account for the outcome being skewed. Correlations between clinical asthma measures and systemic inflammation were assessed using the Spearman rank test $\left(r_{s}\right)$ in subjects with asthma. p-values of $<0.05$ were considered statistically significant.

\section{RESULTS}

\section{Subject characteristics}

Characteristics of study subjects are presented in table 1 . There were no differences in age or sex between groups. Obese and nonobese asthmatic subjects had a similar lung function and asthma severity, while obese asthmatics had a higher inhaled corticosteroid (ICS) dose $(\mathrm{p}=0.003)$. 


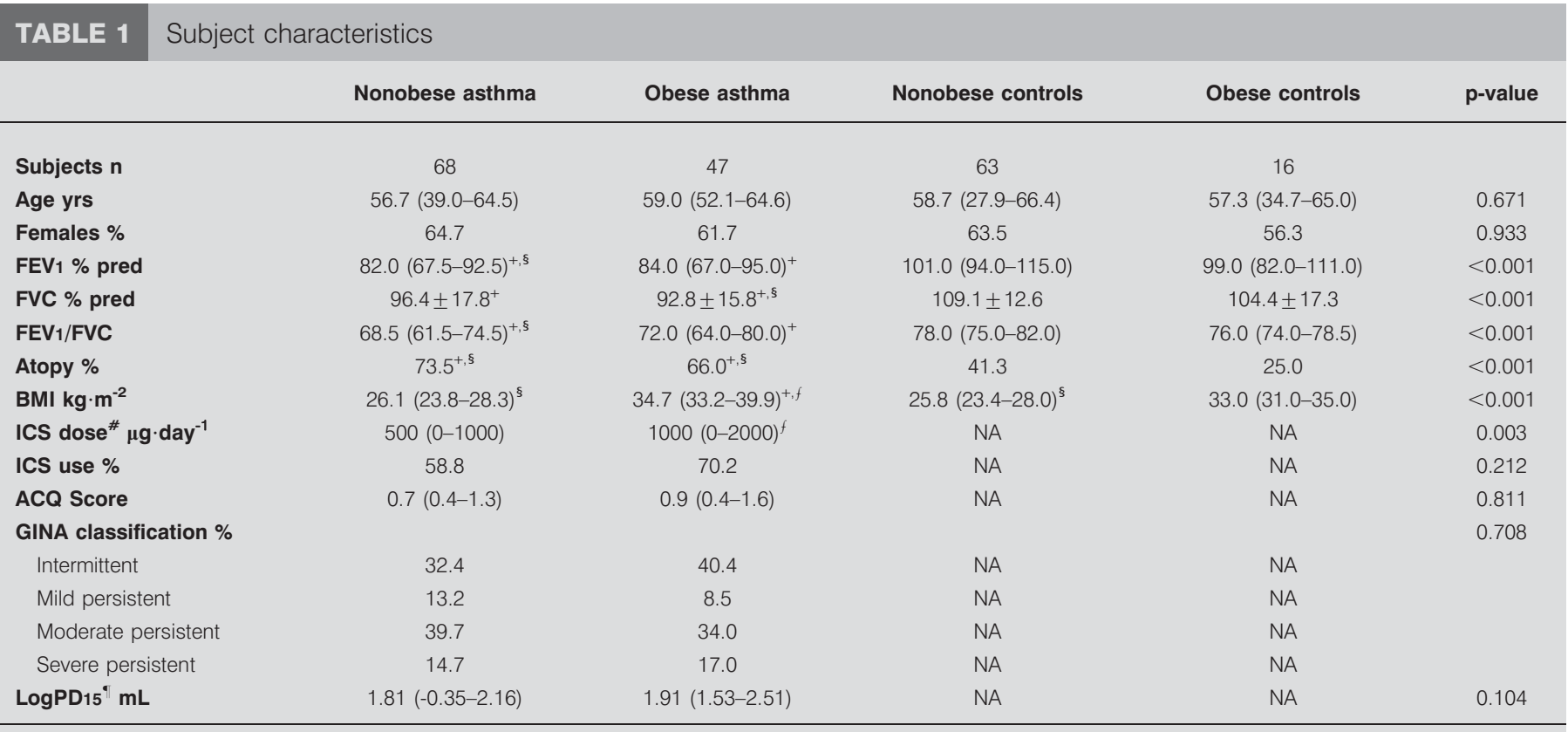

Data are presented as median (interquartile range) or mean $\pm \mathrm{SD}$, unless otherwise stated. FEV1: forced expiratory volume in $1 \mathrm{~s}$; \% pred: \% predicted; FVC: forced vital capacity; BMI: body mass index; ICS: inhaled corticosteroid; ACQ: Asthma Control Questionnaire; GINA: Global Initiative for Asthma; PD15: provocation dose of 4.5\% hypertonic saline required to induce a drop in FEV1 of $15 \%$ from baseline; NA: not available. ${ }^{*}$ : beclomethasone equivalents; " : presented as geometric median (interquartile range); ${ }^{+}: p<0.01$ versus nonobese controls; ${ }^{\text {s: }} p<0.02$ versus obese controls; ${ }^{f}: p<0.01$ versus nonobese asthma.

\section{Systemic inflammation, obesity and asthma}

CRP was elevated in obese compared with nonobese subjects (median (IQR) $5.5(2.0-10.4)$ versus $1.6 \quad(0.8-4.2) \mathrm{mg} \cdot \mathrm{L}^{-1}$; $\mathrm{p}<0.0001)$. The results of two-factor ANOVA identified a significant interaction between obesity and asthma on CRP, such that the presence of asthma further increased CRP levels $(p=0.003)$ (table 2). CRP was significantly higher in obese asthma compared with obese controls $(p=0.008)$ (fig. 1a). There was also a significant effect of asthma on IL-6 $(\mathrm{p}=0.044)$ and an effect of obesity on leptin $(\mathrm{p}<0.001)($ table 2$)$. IL-6 was significantly elevated in obese asthma compared to both obese controls $(p=0.013)$ and nonobese asthma $(p<0.0001)$ (fig. 1b). Leptin was significantly elevated in obese asthma compared with nonobese asthma and nonobese controls, while there was a nonsignificant trend towards increased leptin in obese asthma compared with obese controls $(p=0.109)$ (fig. 1c). IL-6 was negatively correlated with FEV1 and FVC $\left(r_{s}=-0.376, p<0.001\right.$ and $r_{s}=-0.515, p<0.001$, respectively; $\mathrm{n}=112)$ and positively with ACQ score $\left(\mathrm{r}_{\mathrm{s}}=\right.$ $0.275, \mathrm{p}=0.003 ; \mathrm{n}=111)$. In asthmatic subjects, males had a significantly higher IL-6 (median (IQR) 1.9 (1.3-3.0) versus 1.4 $\left.(1.1-2.4) \mathrm{pg} \cdot \mathrm{mL}^{-1} ; \mathrm{p}=0.036\right)$, and reduced CRP (2.2 (0.9-5.6) versus $\left.3.5(1.3-9.6) \mathrm{mg} \cdot \mathrm{L}^{-1} ; \mathrm{p}=0.069\right)$ and leptin $(3,038(2,055-$ $5,906)$ versus $\left.8,205(3,539-11,199) \mathrm{pg} \cdot \mathrm{mL}^{-1} ; \mathrm{p}<0.001\right)$ compared with females.

\section{Airway inflammation, obesity and asthma}

Sputum neutrophil percentages were significantly elevated in obese asthmatic compared with nonobese asthmatic subjects $(\mathrm{p}=0.005)$ and obese controls $(\mathrm{p}=0.001)$ (fig. $2 \mathrm{a})$. Sputum eosinophil percentages were significantly elevated in obese and nonobese asthma, compared with nonobese controls $(\mathrm{p}=0.007$ and $\mathrm{p}<0.0001$, respectively) (fig. $2 \mathrm{~b}$ ). There was a nonsignificant trend towards increased sputum eosinophil percentage in obese asthmatics compared with obese healthy controls $(p=0.275)$.

The results of two-factor ANOVA show an interaction between obesity and asthma resulting in increased sputum neutrophil percentages $(\mathrm{p}=0.012)$ (table 2$)$. There was a significant positive effect of asthma on sputum eosinophil percentages $(p=0.001)$; however, there was no effect of obesity $(p=0.157)$ (table 2). There was a significant interaction between asthma and obesity, resulting in decreased levels of absolute macrophages $(p=0.032)$ (table 2). There was a trend towards an increased absolute neutrophil count in obese asthma compared with nonobese asthma $(p=0.350)$ and nonobese controls $(p=0.159)$. There were weak correlations between neutrophilic airway inflammation and CRP $\left(\mathrm{r}_{\mathrm{s}}=0.283, \mathrm{p}=0.005\right)$ and IL-6 $\left(r_{s}=0.284, p=0.005\right)$ in subjects with asthma. Sputum IL-8 was measured in a subset of patients $(n=82)$, with no significant difference between nonobese and obese asthma (mean (IQR) 6.7 (3.5-14.9) versus $\left.6.9(3.1-12.4) \mathrm{ng} \cdot \mathrm{mL}^{-1} ; \mathrm{p}=0.887\right)$. In females alone, there was a small but nonsignificant increase in IL-8 in obese compared with nonobese asthma (6.4 (2.5-9.8) versus 4.8 (3.0-7.6) $\left.\mathrm{ng} \cdot \mathrm{mL}^{-1} ; \mathrm{p}=0.655\right)$.

In a multiple linear regression model, sputum neutrophil percentages and BMI were positively associated in subjects with asthma ( $\beta$-coefficient (95\% CI) $0.801 \quad(0.102-1.499)$; $\mathrm{p}=0.025$ ) (table 3). Interestingly, this relationship was strengthened when looking at females alone ( $\beta$-coefficient $(95 \% \mathrm{CI})$ $1.015(0.258-1.772) ; \mathrm{p}=0.009)$ (table 4 ) but was not significant in males $(p=0.594)$ (table 5). In females, for every unit increase in BMI there was a corresponding increase in neutrophil 


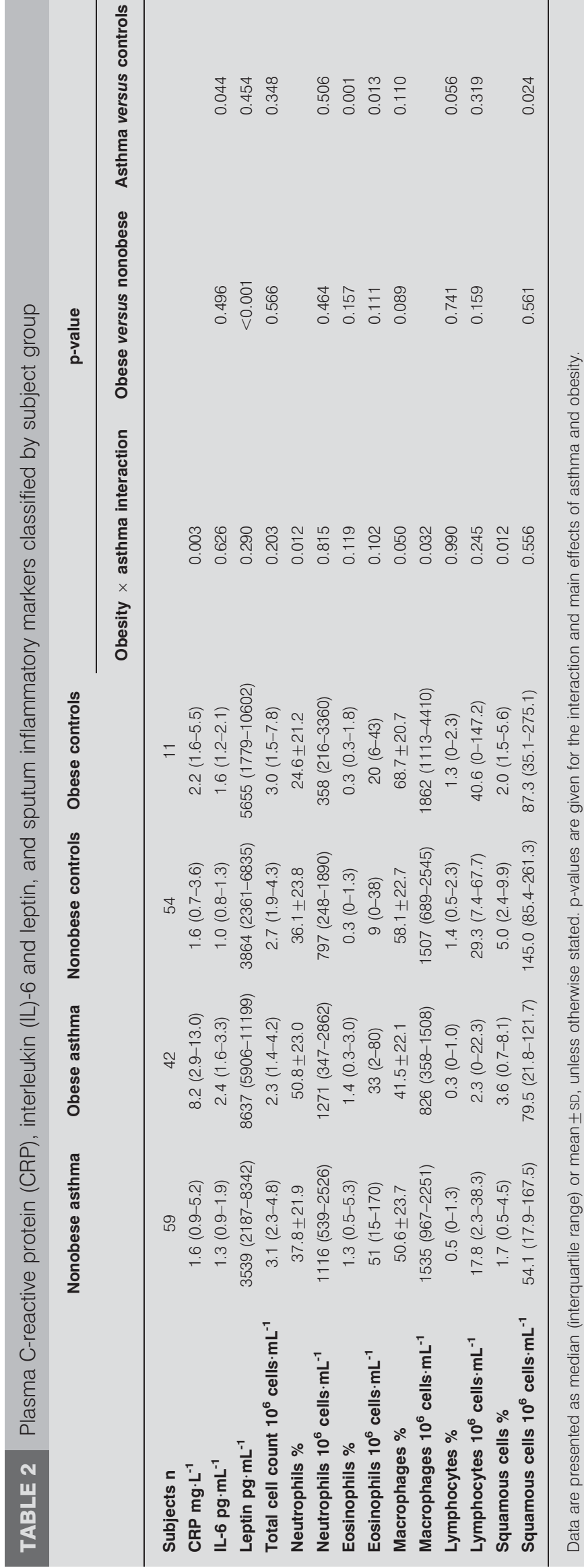

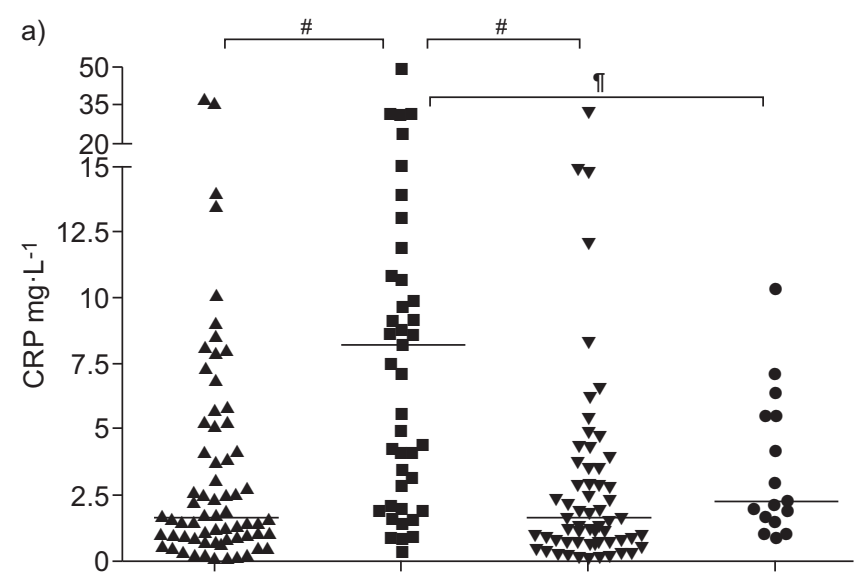
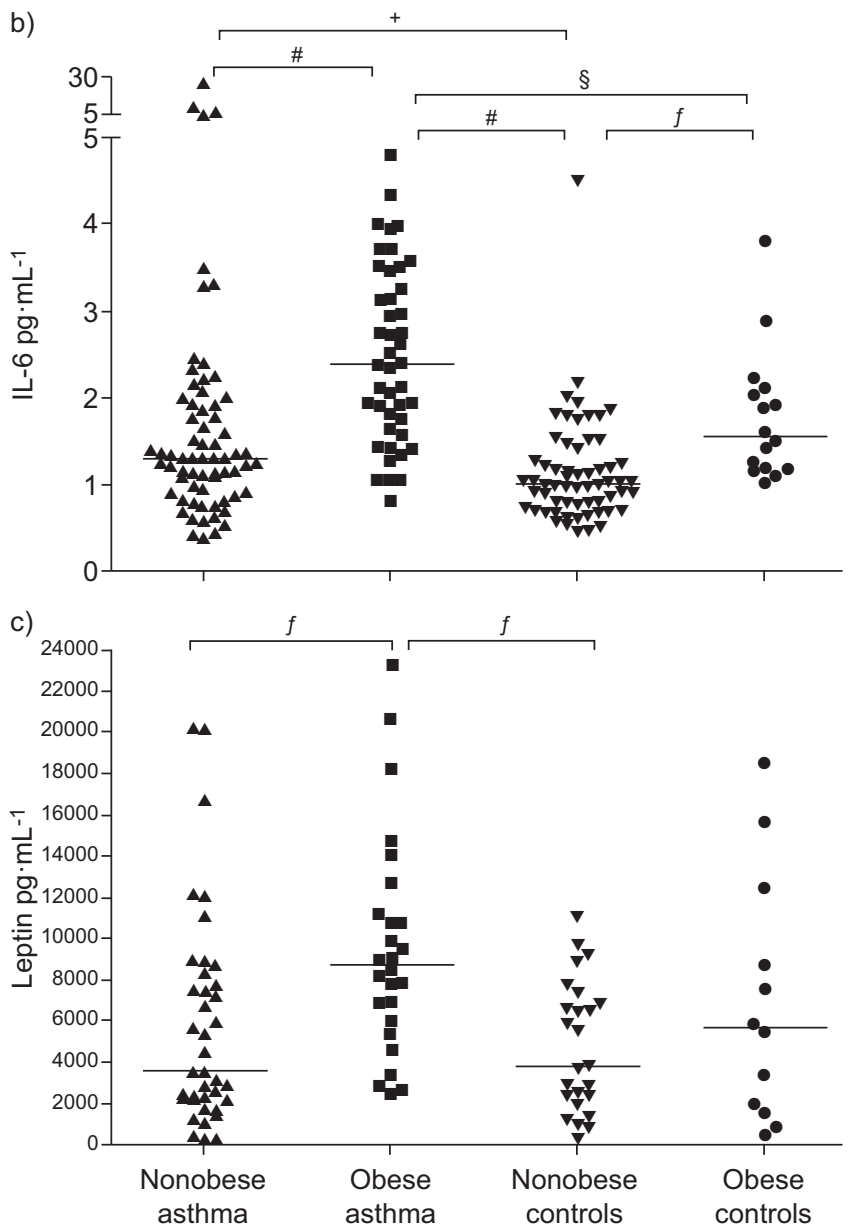

FIGURE 1. a) C-reactive protein (CRP) concentration, b) interleukin (IL)-6 concentration and c) leptin concentration in nonobese and obese subjects with and without asthma. Horizontal bars represent median concentrations. ${ }^{*}: p<0.0001$; $\because: p=0.008 ;^{+}: p=0.003 ;{ }^{s}: p=0.013 ;{ }^{f}: p=0.001$.

percentage of $1.0 \%$. There was no association between sputum neutrophil percentage and BMI in healthy controls $(p=0.828$, $n=65$ ), or between neutrophil percentage and ICS dose in any of the regression models conducted (tables 3-5). Neutrophilic asthma (airway neutrophils $\geqslant 61 \%$ ) was present in a greater proportion of obese than of nonobese asthmatic females $(42.9 \%$ versus $16.2 \% ; \mathrm{p}=0.017, \mathrm{n}=65)$, while obese and nonobese 

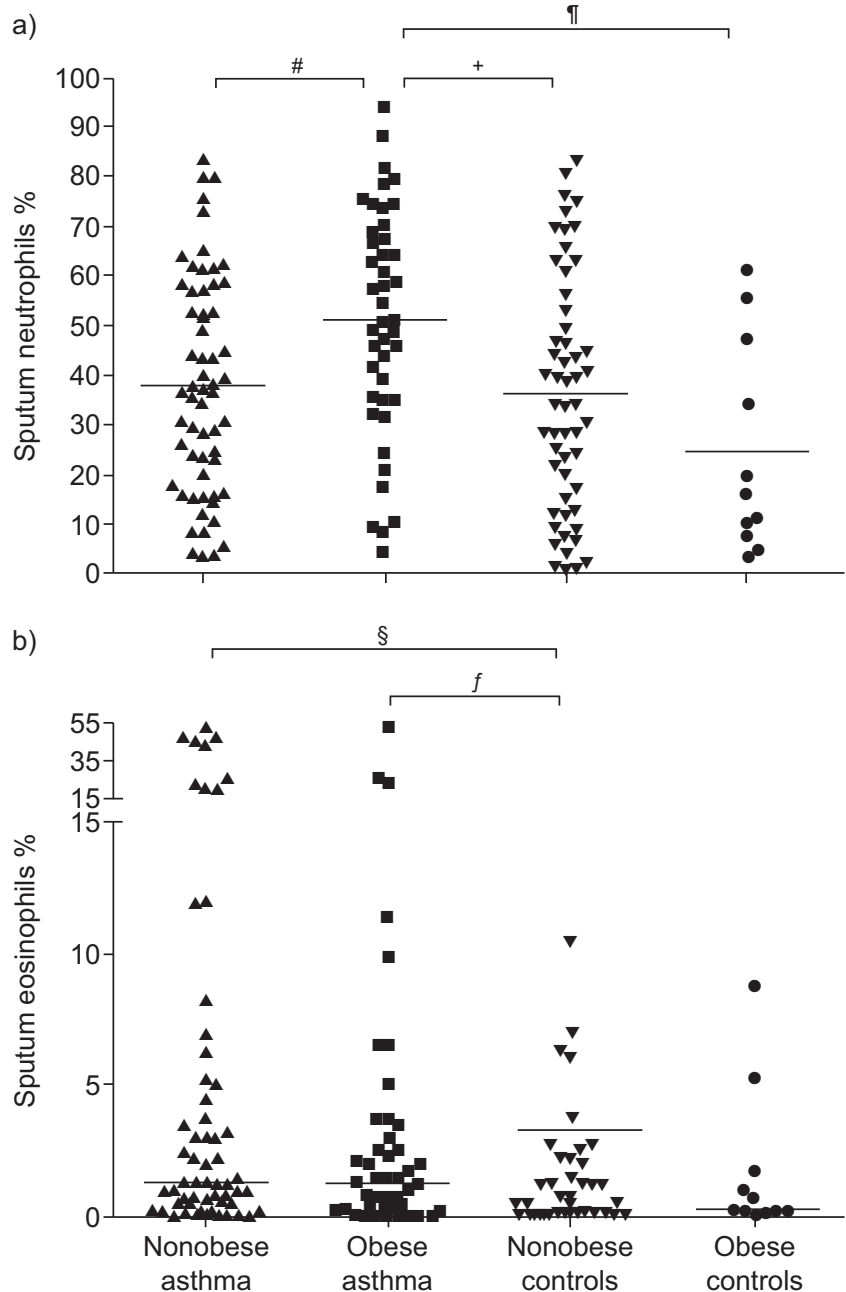

FIGURE 2. a) Sputum neutrophil percentages and b) sputum eosinophil percentages in nonobese and obese subjects with and without asthma. Horizontal bars represent a) mean and b) median concentrations. ${ }^{*}: p=0.005 ; ": p=0.001$; ${ }^{+}: p=0.003 ;{ }^{s}: p<0.0001 ; f: p=0.007$. asthmatic males had similar levels $(28.6 \%$ versus $22.8 \%$; $p=0.693, n=36$ ) (fig. 3). There was no relationship between plasma leptin and sputum neutrophil percentage $(p=0.618)$ or sputum eosinophils percentage $(p=0.432)$ in subjects with asthma.

\section{Airway inflammation and plasma fatty acids in asthma}

Plasma saturated fatty acid percentages were significantly higher in obese males $(30.4 \pm 2.4 \%)$ compared with nonobese males and females and obese females $(28.6 \pm 1.7 \%, 28.6 \pm 1.7 \%$ and $28.8 \pm 1.4 \%$, respectively; $\mathrm{p}<0.02$ for all) (table 6). Obese males also had higher proportions of plasma monounsaturated fatty acids $(29.2 \pm 3.1 \%)$, compared with nonobese males and females and obese females $(25.2 \pm 2.8 \%, 23.9 \pm 3.6 \%$ and $26.3 \pm 2.7 \%$, respectively; $\mathrm{p}<0.02$ for all).

Fatty acids were not a predictor of sputum neutrophil percentages in the multivariate regression model including all subjects with asthma (table 3 ). However, when stratified by sex, asthmatic males showed a positive association between total plasma saturated fatty acids and sputum neutrophil percentages ( $\beta$-coefficient (95\% CI) $0.108(0.036-0.180) ; p=0.004)$, and a negative association between monounsaturated fatty acids and sputum neutrophil percentages ( $\beta$-coefficient $(95 \% \mathrm{CI})-0.068$ $(-0.131--0.005) ; p=0.035)$ (table 5). No relationship between polyunsaturated fatty acids and sputum neutrophil percentages was present in any of the multivariate models (tables 3-5). Interestingly there was no association between sputum neutrophil percentages and fatty acids in females with asthma (table 4).

\section{DISCUSSION}

This was the first study to demonstrate an increase in neutrophilic airway inflammation in obese compared with nonobese asthmatics, and to examine its relationship to circulating fatty acids. The inflammatory effect of obese asthma appears to occur via innate immune pathways, as we observed a significant increase in the proportion of neutrophils in the airways of obese asthmatics and a significant elevation of circulating IL-6. Interestingly, when subjects were stratified by sex, this relationship was only significant in asthmatic females. In males, we found that plasma saturated fatty acid percentages

TABLE 3 Multiple linear regression model describing predictors of sputum neutrophil percentages in all subjects with asthma

\begin{tabular}{|c|c|c|c|c|}
\hline & \multicolumn{2}{|c|}{ Unadjusted model } & \multicolumn{2}{|c|}{ Final model $\left(R^{2}=0.108, p=0.004\right)$} \\
\hline Sex & $0.108(-9.486-9.702)$ & 0.982 & & \\
\hline$B M I \mathbf{k g} \cdot \mathrm{m}^{-2}$ & $0.937(0.238-1.635)$ & 0.009 & $0.801(0.102-1.499)$ & 0.025 \\
\hline Total SFA mg $\cdot \mathrm{L}^{-1}$ & $0.017(-0.002-0.036)$ & 0.075 & & \\
\hline ICS dose $^{\#} \mu \mathrm{g} \cdot$ day $^{-1}$ & $0.003(-0.002-0.009)$ & 0.235 & & \\
\hline Use of ICS treatment yes/no & $4.831(-4.515-14.177)$ & 0.308 & & \\
\hline
\end{tabular}

BMI: body mass index; SFA: saturated fatty acids; MUFA: monounsaturated fatty acids; PUFA: polyunsaturated fatty acids; ICS: inhaled corticosteroid

\#: beclomethasone equivalents. 
TABLE 4 Multiple linear regression model describing predictors of sputum neutrophil percentages in females with asthma

\begin{tabular}{|c|c|c|c|c|}
\hline & \multicolumn{2}{|c|}{ Unadjusted model } & \multicolumn{2}{|c|}{ Final model $\left(R^{2}=0.102, p=0.009\right)$} \\
\hline & $\beta$-coefficient $(95 \% \mathrm{Cl})$ & $\mathrm{p}$-value & $\beta$-coefficient $(95 \% \mathrm{Cl})$ & p-value \\
\hline Age yrs & $0.419(0.025-0.814)$ & 0.037 & & \\
\hline $\mathrm{BMI} \mathbf{k g} \cdot \mathrm{m}^{-2}$ & $1.015(0.258-1.772)$ & 0.009 & $1.015(0.258-1.772)$ & 0.009 \\
\hline Total SFA mg $\cdot \mathrm{L}^{-1}$ & $0.003(-0.023-0.028)$ & 0.840 & & \\
\hline ICS dose ${ }^{\#} \mu \mathrm{g} \cdot$ day $^{-1}$ & $0.004(-0.002-0.011)$ & 0.173 & & \\
\hline Use of ICS treatment yes/no & $8.508(-3.147-20.163)$ & 0.150 & & \\
\hline
\end{tabular}

BMI: body mass index; SFA: saturated fatty acids; MUFA: monounsaturated fatty acids; PUFA: polyunsaturated fatty acids; ICS: inhaled corticosteroid.

\#: beclomethasone equivalents.

were a positive predictor of neutrophil percentage. This suggests that in asthmatic females body fat may be a key driver of airway inflammation.

Female sex dominance is central to the obese-asthma phenotype proposed by HALDAR et al. [6] and is supported by several epidemiological studies. Our results demonstrate a positive relationship between BMI and neutrophil percentage in females. This may be explained, at least in part, by leptin. Leptin is secreted 2-3 times more highly from subcutaneous than from visceral adipose tissue and correlates more strongly with adiposity in females $[12,17]$. Leptin promotes T-helper type 1 cell differentiation and increases activation of neutrophils via tumour necrosis factor (TNF)- $\alpha$ [18]. Indeed, in a mouse pneumococcal pneumonia model, exogenous leptin stimulated the innate immune response leading to increased neutrophils and IL-6 in bronchoalveolar lavage fluid [19]. Because leptin is strongly related to adiposity in females, the relationship between circulating neutrophils and BMI would be expected to follow a similar pattern. It is plausible that this inflammatory effect extends to the airways of subjects with asthma. Interestingly, we did not observe a relationship between plasma leptin and airway neutrophils; however, assessment of airway leptin levels may provide more insight. BRUNO et al. [20] observed a differential expression of leptin in both the submucosa and epithelial cells in the airways of people with chronic obstructive pulmonary disease (COPD) compared with both healthy controls and smokers, while COPD severity was associated with the presence of these leptin-positive cells. The importance of leptin is still being investigated in asthma; a relationship between circulating leptin levels and risk of asthma development was observed in females in one study [21], while another cited no relationship [22]. We observed only a very small difference in sputum IL- 8 between obese and nonobese asthmatic females, which is unlikely to be relevant, suggesting another mediator is responsible for the association between sputum neutrophils and BMI.

Few studies have examined the relationship between dietary fat and asthma. Saturated fatty acids activate the innate immune response via Toll-like receptor (TLR)4 and TLR2, leading to activation of nuclear factor (NF)- $\mathrm{\kappa B}$ and upregulation of the pro-inflammatory cytokines TNF- $\alpha$, IL- 6 and IL- 8 [23]. Monounsaturated fatty acids have been shown to inhibit this pathway [24]. The current study observed a relationship between saturated and monounsaturated fatty acids, and neutrophilic airway inflammation, in males only. This may be explained by sex differences in fatty acid storage and

TABLE 5 Multiple linear regression model describing predictors of sputum neutrophil percentages in males with asthma

\begin{tabular}{|c|c|c|c|c|}
\hline & \multicolumn{2}{|c|}{ Unadjusted model } & \multicolumn{2}{|c|}{ Final model $\left(R^{2}=0.286, p=0.005\right)$} \\
\hline & $\beta$-coefficient $(95 \% \mathrm{Cl})$ & p-value & $\beta$-coefficient $(95 \% \mathrm{Cl})$ & p-value \\
\hline Age yrs & $0.423(-0.158-1.003)$ & 0.148 & & \\
\hline BMI $\mathrm{kg} \cdot \mathrm{m}^{-2}$ & $0.594(-1.208-2.397)$ & 0.507 & & \\
\hline Total SFA $\mathrm{mg} \cdot \mathrm{L}^{-1}$ & $0.036(0.008-0.065)$ & 0.014 & $0.108(0.036-0.180)$ & 0.004 \\
\hline ICS $_{\text {dose }}^{\#} \mu \mathrm{g} \cdot$ day $^{-1}$ & $0.000(-0.012-0.012)$ & 0.962 & & \\
\hline Use of ICS treatment yes/no & $-1.339(-17.900-15.222)$ & 0.870 & & \\
\hline
\end{tabular}

BMI: body mass index; SFA: saturated fatty acids; MUFA: monounsaturated fatty acids; PUFA: polyunsaturated fatty acids; ICS: inhaled corticosteroid

\#: beclomethasone equivalents. 


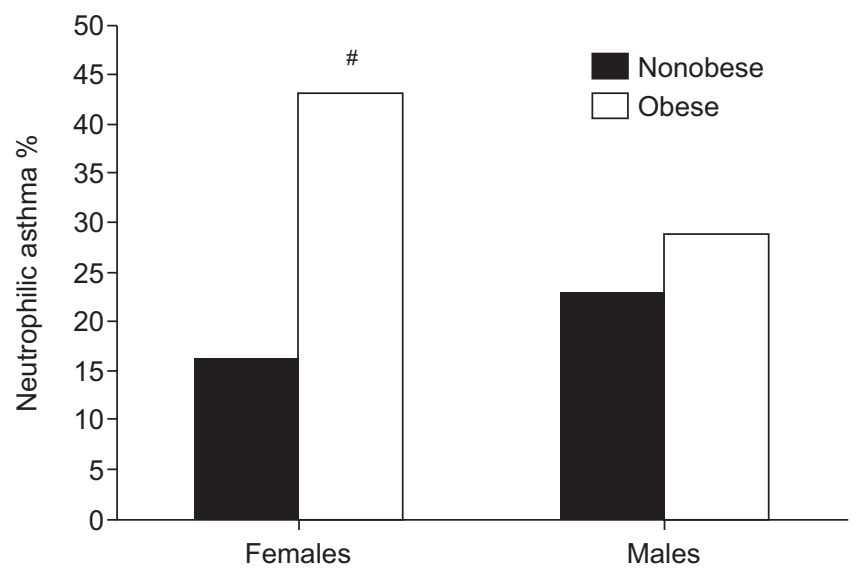

FIGURE 3. Increased proportion of neutrophilic asthma (sputum neutrophils $\geqslant 61 \%$ ) in obese females with asthma. ${ }^{*}: p=0.017$ versus nonobese asthmatic females.

release. Females are more likely to store fatty acids, with an increased post-prandial fat deposition in the gluteal-femoral region compared with males [25]. Males, on the other hand, have a 6-7-fold higher postprandial fatty acid uptake in the visceral abdominal depot and a 1.5-3-fold higher rate of lipolysis in the same region compared with BMI- and agematched females [13]. Most fatty acids released from the visceral abdominal depot are transported via the portal circulation directly to the liver [26]. Kupffer cells are hepaticresident macrophages that are potently activated by saturated fatty acids via the TLR4 pathway, leading to the release of proinflammatory cytokines such as IL-6 [26]. The significantly larger flux of fatty acids being transported to the liver in males could result in a greater sensitivity to fatty acid-induced inflammation and thus explain the sex bias in the current study. The relationship between saturated fatty acids and neutrophil percentages, which is independent of BMI, is noteworthy because airway neutrophils are clinically important in asthma. The most severe forms of asthma involve elevated neutrophils [27], and sputum neutrophils negatively correlate with lung function (\% predicted FEV1) and airflow obstruction in asthma [5]. Thus, stimuli that lead to increased airway neutrophilia are relevant to clinical asthma outcomes.

Interestingly, we did not observe a relationship between BMI and fatty acid levels. Obesity results from excessive caloric intake, which may result from excess fat intake but has also been very strongly linked with excess carbohydrate intake [28], which would have no direct effect on plasma fatty acid levels. This may account for the lack of association between BMI and plasma fatty acids in this study.

An increase in systemic inflammation in obesity has been well described. However, its relationship with airway inflammation is less clear. It is plausible that systemic inflammation does affect the airways. Indeed genetically IL-6-deficient, ozoneexposed mice have reduced airway neutrophils [29], while elevated CRP is associated with accelerated lung function decline independent of BMI [30]. In this study, asthma independently increased plasma levels of IL- 6 by $\sim 50 \%$. Plasma IL-6 was negatively correlated with FEV1 and forced vital capacity, and positively with sputum neutrophils and ACQ score in asthma, suggesting that this increase in systemic inflammation affects the airways. While the level of IL-6 we observed was quite low, it is still of physiological importance in the airways due to its downstream effects. Indeed SUNYER et al. [31] observed a negative association between IL-6 and lung function in 134 subjects post-myocardial infarction, who had similarly low levels of IL-6.

While the obese and nonobese asthmatics in this study had similar lung function, the obese subjects were taking a higher dose of ICS. Obese asthmatics have been observed to be resistant to steroid treatment [32]. It is plausible that this steroid resistance is related to obese subjects having a more neutrophilic pattern of inflammation, as neutrophilic asthmatics have also been shown to be steroid resistant [33]. In a recent steroid response trial in asthma, CowAN et al. [34] observed an $8.4 \%$ increase in sputum neutrophils after $\geqslant 28$ days of fluticasone treatment. In the present study, obese subjects were indeed taking a higher ICS dose than their nonobese counterparts. However, this appears to have had minimal effect on the overall pattern of inflammation, as ICS dose was not a predictor of airway neutrophilia in the regression analyses conducted.

A limitation of this study is that body fat distribution was not measured, though sex differences in body fat proportions and distribution were accounted for by examining males and females separately. Another limitation is the cross-sectional study design. In order to verify a causal role for obesity and dietary fat independently driving neutrophilic inflammation, studies of weight change and dietary fat modification are

TABLE 6 Total and percentage plasma fatty acids (FAs) in nonobese and obese subjects with asthma, by sex

\begin{tabular}{|c|c|c|c|c|c|}
\hline & $\begin{array}{c}\text { Nonobese asthmatic } \\
\text { females }\end{array}$ & $\begin{array}{c}\text { Nonobese asthmatic } \\
\text { males }\end{array}$ & $\begin{array}{l}\text { Obese asthmatic } \\
\text { females }\end{array}$ & $\begin{array}{c}\text { Obese asthmatic } \\
\text { males }\end{array}$ & p-value \\
\hline Total FAs $\mathrm{mg} \cdot \mathrm{L}^{-1}$ & 3316 (2795-3898) & 2966 (2612-3335) & 3232 (2924-3586) & $3385(3034-4400)$ & 0.150 \\
\hline$\%$ SFA & $28.6 \pm 1.7^{\#}$ & $28.6 \pm 1.7^{\#}$ & $28.8 \pm 1.4^{\#}$ & $30.4 \pm 2.4$ & 0.005 \\
\hline$\%$ MUFA & $23.9 \pm 3.6^{\#, \star \star \star}$ & $25.2 \pm 2.8^{\#}$ & $26.3 \pm 2.7^{\#}$ & $29.2 \pm 3.1$ & $<0.001$ \\
\hline$\%$ PUFA & $47.5 \pm 4.6^{\#}$ & $46.3 \pm 3.5^{\#}$ & $44.9 \pm 3.2^{\#}$ & $40.4 \pm 4.7$ & $<0.001$ \\
\hline
\end{tabular}

Data are presented as median (interquartile range) or mean \pm SD, udless otherwise stated. SFA: saturated fatty acids; MUFA: monounsaturated fatty acids; PUFA: polyunsaturated fatty acids. 
needed. A limited number of studies have examined the effect of weight loss in asthma and have generally demonstrated improvements to respiratory symptoms. Preliminary results from a study by DixON et al. [35] included 16 morbidly obese asthmatic subjects who underwent bariatric surgery. Significant improvements in lung function and asthma control were observed, with no effect on airway inflammation. Interestingly, this is the only weight loss trial to have examined airway inflammation in asthma. No published intervention studies have examined the effect of dietary fat on neutrophilic airway inflammation.

In conclusion, we have shown for the first time an increase in neutrophilic airway inflammation in obese compared with nonobese asthmatics. When stratified by sex, this relationship was only significant in asthmatic females. In asthmatic males, saturated fatty acids were associated with increased neutrophilic airway inflammation and monounsaturated fats with reduced neutrophilic airway inflammation. Our data suggests that both obesity and saturated fat may independently activate innate immune responses, leading to a more neutrophilic pattern of airway inflammation, with distinct differences between males and females. This is an important development in understanding the link between obesity, dietary fat, inflammation and asthma. Neutrophilic inflammation is a relevant mechanism of the obese asthma phenotype in females and it is likely that treatment strategies that target males and females differentially will need to be developed. This study also suggests that future research examining the obese asthma phenotype will need to adjust for sex effects. Future research should include interventions examining the effects of weight loss, dietary fat modification, body composition and sex on adipokines, systemic and airway inflammation, and clinical asthma markers. This will confirm the role of obesity and dietary fat in driving airway neutrophilia and thus worsening asthma outcomes.

\section{SUPPORT STATEMENT}

This research was supported by a National Health and Medical Research Council (Canberra, Australia) Centre for Clinical Research Excellence postgraduate scholarship and a Hunter Medical Research Institute (Newcastle, Australia) postgraduate student support package sponsored by the Greaves family.

\section{STATEMENT OF INTEREST}

A statement of interest for P.G. Gibson can be found at www.erj. ersjournals.com/site/misc/statements.xhtml

\section{ACKNOWLEDGEMENTS}

The authors thank J. Smart and the Hunter Medical Research Institute Respiratory Research laboratory staff for their assistance with data acquisition, and P. Mcelduff (Hunter Medical Research Institute, John Hunter Hospital, Newcastle, Australia) and H. Powell (Respiratory and Sleep Medicine, Hunter Medical Research Institute) for statistical advice.

\section{REFERENCES}

1 Shore SA. Obesity and asthma: possible mechanisms. J Allergy Clin Immunol 2008; 121: 1087-1093.

2 Lessard A, Turcotte H, Cormier Y, et al. Obesity and asthma: a specific phenotype? Chest 2008; 134: 317-323.
3 Todd DC, Armstrong S, D'Silva L, et al. Effect of obesity on airway inflammation: a cross-sectional analysis of body mass index and sputum cell counts. Clin Exp Allergy 2007; 37: 1049-1054.

4 Sutherland TJT, Cowan JO, Young S, et al. The association between obesity and asthma: interactions between systemic and airway inflammation. Am J Respir Crit Care Med 2008; 178: 469-475.

5 Simpson JL, Scott RJ, Boyle MJ, et al. Inflammatory subtypes in asthma: assessment and identification using induced sputum. Respirology 2006; 11: 54-61.

6 Haldar P, Pavord ID, Shaw DE, et al. Cluster analysis and clinical asthma phenotypes. Am J Respir Crit Care Med 2008; 178: 218-224.

7 Nijhuis J, Rensen SS, Slaats Y, et al. Neutrophil activation in morbid obesity, chronic activation of acute inflammation. Obesity 2009; 17: 2014-2018.

8 Shah TJ, Leik CE, Walsh SW. Neutrophil infiltration and systemic vascular inflammation in obese women. Reprod Sci 2010; 17: 116-124.

9 Wood LG, Scott HA, Garg ML, et al. Innate immune mechanisms linking non-esterified fatty acids and respiratory disease. Prog Lipid Res 2009; 48: 27-43.

10 Ólafsdottir IS, Gislason T, Thjodleifsson B, et al. C reactive protein levels are increased in non-allergic but not allergic asthma: a multicentre epidemiological study. Thorax 2005; 60: 451-454.

11 Chen Y, Dales R, Krewski D, et al. Increased effects of smoking and obesity on asthma among female Canadians: the National Population Health Survey, 1994-1995. Am J Epidemiol 1999; 150: 255-262.

12 Woods SC, Gotoh K, Clegg DJ. Gender differences in the control of energy homeostasis. Exp Biol Med 2003; 228: 1175-1180.

13 Shi H, Clegg DJ. Sex differences in the regulation of body weight. Physiol Behav 2009; 97: 199-204.

14 Juniper EF, O'Byrne PM, Guyatt GH, et al. Development and validation of a questionnaire to measure asthma control. Eur Respir J 1999; 14: 902-907.

15 Gibson PG, Wlodarczyk J, Hensley M, et al. Epidemiological association of airway inflammation with asthma symptoms and airway hyperresponsiveness in childhood. Am J Respir Crit Care Med 1998; 158: 36-41.

16 Lepage G, Roy CC. Direct transesterification of all classes of lipid in a one-step reaction. J Lipid Res 1986; 27: 114-120.

17 Van Harmelen V, Reynisdottir S, Eriksson P, et al. Leptin secretion from subcutaneous and visceral adipose tissue in women. Diabetes 1998; 47: 913-917.

18 Zarkesh-Esfahani H, Pockley AG, Wu Z, et al. Leptin indirectly activates human neutrophils via induction of TNF- $\alpha$. J Immunol 2004; 172: 1809-1814.

19 Mancuso P, Huffnagle GB, Olszewski MA, et al. Leptin corrects host defense defects after acute starvation in murine pneumococcal pneumonia. Am J Respir Crit Care Med 2006; 173: 212-218.

20 Bruno A, Chanez P, Chiappara G, et al. Does leptin play a cytokine-like role within the airways of COPD patients? Eur Respir J 2005; 26: 398-405.

21 Sood A, Ford ES, Camargo CA. Association between leptin and asthma in adults. Thorax 2006; 61: 300-305.

22 Sutherland TJT, Sears MR, McLachlan CR, et al. Leptin, adiponectin, and asthma: findings from a population-based cohort study. Ann Allergy Asthma Immunol 2009; 103: 101-107.

23 Lee JY, Zhao L, Youn HS, et al. Saturated fatty acid activates but polyunsaturated fatty acid inhibits Toll-like receptor 2 dimerized with Toll-like receptor 6 or 1. J Biol Chem 2004; 279: 16971-16979.

24 Lee JY, Plakidas A, Lee WH, et al. Differential modulation of Tolllike receptors by fatty acids: preferential inhibition by $\mathrm{n}-3$ polyunsaturated fatty acids. J Lipid Res 2003; 44: 479-486.

25 Power ML, Schulkin J. Sex differences in fat storage, fat metabolism, and the health risks from obesity: possible evolutionary origins. Br J Nutr 2008; 99: 931-940.

26 de Luca C, Olefsky JM. Inflammation and insulin resistance. FEBS Lett 2008; 582: 97-105. 
27 Ordonez CL, Shaughnessy TE, Matthay MA, et al. Increased neutrophil numbers and IL-8 levels in airway secretions in acute severe asthma. Am J Respir Crit Care Med 2000; 161: 1185-1190.

28 Ludwig DS, Peterson KE, Gortmaker SL. Relation between consumption of sugar-sweetened drinks and childhood obesity: a prospective, observational analysis. Lancet 2001; 357: 505508.

29 Johnston RA, Schwartzman IN, Flynt L, et al. Role of interleukin-6 in murine airway responses to ozone. Am J Physiol Lung Cell Mol Physiol 2005; 288: L390-L397.

30 Rasmussen F, Mikkelsen D, Hancox RJ, et al. High-sensitive Creactive protein is associated with reduced lung function in young adults. Eur Respir J 2009; 33: 382-388.
31 Sunyer J, Pistelli R, Plana E, et al. Systemic inflammation, genetic susceptibility and lung function. Eur Respir J 2008; 32: 92-97.

32 Boulet L-P, Frannsen E. Influence of obesity on response to fluticasone with or without salmeterol in moderate asthma. Respir Med 2007; 101: 2240-2247.

33 Green RH, Brightling CE, Woltmann G, et al. Analysis of induced sputum in adults with asthma: identification of subgroup with isolated sputum neutrophils and poor response to inhaled corticosteroids. Thorax 2002; 57: 875-879.

34 Cowan DC, Cowan J, Palmay R, et al. Effects of steroid therapy on inflammatory cell subtypes in asthma. Thorax 2010; 65: 384-390.

35 Dixon AE, Pratley RE, Spaulding L, et al. Pathophysical changes in asthma induced by weight loss. Proc Am Thorac Soc 2009; 6: 328. 